\title{
AVALIAÇÃO ANTROPOMÉTRICA E REGISTRO ALIMENTAR DE UM GRUPO DE IDOSOS INTEGRANTES DE UM PROGRAMA DE SAÚDE EM MINEIROS-GO
}

\section{ARTIGO ORIGINAL}

CARRIJO, Lucilene Santos Pereira ${ }^{1}$, FRANÇA, Ana Paula Souza ${ }^{2}$, TAVARES, Dielly Custódio ${ }^{3}$, OLIVEIRA, Kelly Kristina Nogueira de ${ }^{4}$, SILVA, Istefane Borges da ${ }^{5}$, COSTA, Sílvia Souza Lima ${ }^{6}$, FREITAS, Lunara da Silva ${ }^{7}$

\footnotetext{
${ }^{1}$ Nutricionista, pós-graduada em nutrição clínica pela Faculdade Metropolitana de Ribeirão Preto São Paulo (SP).

${ }^{2}$ Nutricionista, graduada pela Faculdade Morgana Potrich (FAMP), Mineiros - GO Brasil.
}

${ }^{3}$ Nutricionista, graduada pela Faculdade Morgana Potrich (FAMP), Mineiros - GO Brasil.

${ }^{4}$ Nutricionista, pós-graduada em nutrição clínica pela Faculdade Metropolitana de Ribeirão Preto São Paulo (SP).

${ }^{5}$ Nutricionista, graduada pela Faculdade Morgana Potrich (FAMP), Mineiros - GO Brasil.

${ }^{6}$ Enfermeira, graduada pela Faculdade Morgana Potrich (FAMP), Mineiros - GO Brasil

7 Orientadora. Nutricionista, Doutora em Ciências pela Faculdade de Medicina da Universidade de São Paulo (USP), São Paulo - SP. Docente da FAMP - Faculdade Morgana Potrich, Mineiros - GO, Brasil. 
CARRIJO, Lucilene Santos Pereira. Et al. Avaliação Antropométrica E Registro Alimentar De Um Grupo De Idosos Integrantes De Um Programa De Saúde Em Mineiros-GO. Revista Científica Multidisciplinar Núcleo do Conhecimento. Ano 06, Ed. 06, Vol. 04, pp. 162-179. Junho de 2021. ISSN: 2448-0959, Link de acesso: https://www.nucleodoconhecimento.com.br/saude/programa-de-saude,

$\mathrm{DOI}:$

10.32749/nucleodoconhecimento.com.br/saude/programa-de-saude

\section{RESUMO}

Atualmente, o número de idosos vem crescendo de maneira relevante no Brasil e no mundo. Acredita-se que esse aumento esteja relacionado com políticas públicas de saúde, assistência social, previdência e a preocupação por um envelhecimento saudável e ativo. Avaliar o perfil antropométrico e alimentar de idosos integrantes de um programa de saúde em Mineiros-GO. Estudo do tipo observacional descritivo, desenvolvido com idosos (idade $\geq 60$ anos), de ambos os sexos. Os indivíduos foram submetidos à antropometria, sendo avaliados a estatura, peso, circunferência da cintura, da panturrilha, braquial e avaliação da gordura corporal por dobras cutâneas. A avaliação do hábito alimentar foi feita pela análise de 3 registros alimentares em dias da semana não consecutivos, incluindo um de final de semana. Participaram 54 idosos, maioria do sexo feminino (85\%), com idade média de 72,4 \pm 6,6 anos. O IMC indicou que $50 \%$ dos idosos estavam com excesso de peso. A circunferência da cintura indicou risco para doenças cardiovasculares entre mulheres, mas não entre homens. O percentual de gordura esteve adequado para mulheres, porém, aumentado para homens ( $\mathrm{M}$ : média $=33,3 \%, \mathrm{DP}= \pm 5,6 \% ; \mathrm{H}$ : média $=31,2 \%$, DP $=$ $\pm 6,9 \%)$. A avaliação do hábito alimentar mostrou ingestão em macronutrientes adequada e micronutrientes abaixo do recomendado. O presente estudo mostrou que, apesar da adequada ingestão dos macronutrientes, o grupo apresentou excesso de peso e risco elevado para doenças crônicas. Diante disso, sugere-se a realização de mais pesquisas com idosos, a fim de contribuir para um envelhecimento natural e saudável.

Palavras-chave: Envelhecimento, Antropometria, Consumo Alimentar. 


\section{INTRODUÇÃO}

Segundo o Instituto Brasileiro de Geografia e Estatística (IBGE) são considerados idosos os indivíduos com faixa etária igual ou superior a 60 anos. Atualmente o número de idosos vem crescendo de maneira significativa no Brasil e no mundo, sendo que no Brasil a estimativa para 2050 alcançará os 66,5 milhões de pessoas $(29,3 \%)$ nessa faixa etária. Acredita-se que esse aumento esteja relacionado com políticas públicas de saúde, assistência social, previdência e a importância de um envelhecimento saudável e ativo, em que a procura se torna cada vez maior para esse grupo (BRASIL, 2016).

O aumento da expectativa de vida da população, pode estar relacionado a descobertas da cura e tratamento de diversas patologias nos últimos anos. Pode-se observar que a qualidade de vida e a economia também estão diretamente ligadas a esse aumento. $O$ envelhecimento acarreta o surgimento de diversas Doenças Crônicas Não Transmissíveis (DCNT), como diabetes mellitus, hipertensão arterial, dislipidemias e câncer. Com isso, surge a necessidade de maior cuidado com a pessoa idosa, além de investimentos nas áreas de saúde, no intuito de promover o envelhecimento saudável de toda população (MIRANDA; MENDES; SILVA, 2016).

A origem das DCNT está relacionada, dentre outros fatores, com o estilo de vida e hábitos alimentares, pois uma alimentação inadequada pode levar ao desenvolvimento de transtornos, que interferem ao longo da vida. Uma alimentação equilibrada é de extrema importância e auxilia no envelhecimento saudável, que pode ser influenciado tanto pelo estilo de vida, quanto por fatores genéticos. Alimentação saudável juntamente com práticas regulares de atividades físicas são medidas para alcançar um envelhecimento saudável (MARCHIONI; FISBERG, 2009). Além disso, é direito de todos o acesso a uma alimentação de qualidade, em quantidades suficientemente adequada, em condições acessíveis do ponto de vista microbiológica, que atendam às necessidades especiais de cada indivíduo e garanta um envelhecimento saudável (GEUS et al., 2011). 
A avaliação antropométrica é de suma importância no processo de envelhecimento, fase em que o indivíduo apresenta mudanças fisiológicas características da idade. Dentre elas pode-se mencionar alterações na cavidade oral, que dificultam a mastigação, deglutição e percepção das características sensoriais dos alimentos. Além disso, há ainda alterações gastrointestinais, metabólicas e perda de massa muscular, levando à sarcopenia, redução da força física e do gasto energético total com consequente perda da autonomia. A alimentação do idoso é um fator que requer bastante atenção, pois é um período em que ocorrem modificações, sejam nas condições internas de seu corpo ou as relacionadas aos contextos externos do ambiente, que modificam os seus hábitos alimentares. É importante lembrar que a velhice é um processo relacionado a mudanças no comportamento, dada às transformações que ocorrem ao longo do tempo (MIRANDA; MENDES; SILVA, 2016; GEUS et al., 2011).

A sarcopenia é definida como a perda de massa magra, força e função muscular, que pode acometer tanto os homens quanto as mulheres, estando associada ao maior risco de quedas e diminuição da capacidade funcional. Com o envelhecimento, a sarcopenia tende a piorar e está relacionada com o baixo Índice de Massa Corporal (IMC) e estilo de vida sedentário (NETO et al., 2012). Acredita-se que a avaliação antropométrica seja importante para a população idosa por fornecer informações que podem refletir no seu estado de saúde e qualidade de vida. Trata-se de um método rápido, de fácil realização, não invasivo e de baixo custo, executado por meio de instrumentos simples como balanças, fitas métricas e adipômetros. É composta por medidas como peso, estatura, circunferências e dobras cutâneas (PFRIMER; FERRIOLLI, 2008). A avaliação nutricional tem como objetivo verificar a presença de doenças ou distúrbios nutricionais, utilizando medidas adequadas de maneira a colaborar na recuperação e manutenção do estado de saúde do indivíduo (MELLO, 2017).

Sendo assim o objetivo deste trabalho foi avaliar o perfil antropométrico e alimentar de idosos integrantes de um programa de saúde em Mineiros-GO.

\section{MATERIAIS E MÉTODOS}




\subsection{DELINEAMENTO E SUJEITOS DO ESTUDO}

Trata-se de uma pesquisa de delineamento observacional descritivo, que foi desenvolvida com um grupo de idosos participantes de um programa de saúde de uma Instituição de Ensino Superior (IES) de Mineiros -Goiás, no período de agosto a novembro de 2019. O grupo de saúde teve início em fevereiro de 2018, e conta atualmente com a participação de 82 idosos, sendo 10 homens e 72 mulheres. $O$ objetivo principal do programa foi proporcionar ao idoso conhecimentos necessários para alcançar uma velhice digna, com saúde e autonomia. Uma equipe multidisciplinar, composta por alunos e professores de uma Instituição de Ensino Superior, de diversas áreas, conduzem as atividades com os participantes em um espaço apropriado. Dentre as atividades desenvolvidas estão a realização de palestras educativas, dinâmicas, rodas de conversa e atividades físicas. O ciclo de atividades ocorre a cada semestre, duas vezes por semana.

Os sujeitos da pesquisa foram definidos por meio de critérios de inclusão: estar atualmente cadastrado no grupo de saúde, ter idades $\geq 60$ anos, ambos os sexos. Já os critérios de exclusão foram: indivíduos que apresentaram condição física ou mental que pudesse ter impedido sua participação ou aqueles que optaram por não participar.

A pesquisa foi aprovada pelo Comitê de Ética em Pesquisa (CEP) sob o número CAAE 14279919.0.0000.5428, por estar de acordo com a Resolução ํㅜ 466/12, do Conselho Nacional de Saúde, que envolve aspectos éticos de pesquisa com seres humanos.

\subsection{PROCEDIMENTOS}

A pesquisa foi realizada em horários e dias estabelecidos, com a coleta de dados no período de agosto a outubro de 2019, com assinatura do Termo de Consentimento Livre e Esclarecido (TCLE). Foi utilizado um questionário elaborado especificamente para esta pesquisa, contendo informações sociodemográficas como data de nascimento, gênero, raça/etnia, situação conjugal, ocupação, renda familiar, prática de atividade física regular, tabagismo, etilismo e diagnóstico de diabetes e hipertensão. 
Após o preenchimento desse questionário, os indivíduos foram submetidos à antropometria, quando foram registradas as medidas de estatura, peso, (para cálculo do IMC), circunferência da cintura, da panturrilha, do braço, muscular do braço e as dobras cutâneas: tricipital, subescapular, panturrilha e suprailíaca.

A estatura foi mensurada por um estadiômetro, com precisão de $1 \mathrm{~mm}$. A medida foi realizada com os indivíduos descalços, em posição ereta, pés unidos, glúteos, ombros e calcanhares encostados na parede, com os braços soltos ao longo do corpo. A determinação da estatura foi feita de acordo com o sexo, baseada nas fórmulas propostas por Chumlea (1985) (CHUMLEA; ROCHE; STEINBAUGH, 1985).

O peso foi verificado por meio de uma balança digital da marca $O m r o n^{\circledR}$ com capacidade para 150 kg, com o indivíduo em posição ereta, descalço, utilizando roupas leves, pés unidos e peso distribuídos em ambos os pés. O IMC foi calculado considerando-se a razão entre a massa corporal (em kg) e a estatura (em metros) ao quadrado $\left(\mathrm{kg} / \mathrm{m}^{2}\right)$ e classificado segundo os pontos de corte propostos por Lipschitz (1994). (Tabela 1).

Tabela 1- Classificação do estado nutricional segundo IMC para idosos.

\begin{tabular}{|l|l|}
\hline IMC $\left(\mathrm{Kg} / \mathrm{m}^{2}\right)$ & \multicolumn{1}{|c|}{ Classificação } \\
\hline$<\mathbf{2 2}$ & Baixo peso \\
\hline $\mathbf{2 2 - 2 7}$ & Eutrofia \\
\hline $\mathbf{2} \mathbf{2 7}$ & Excesso de peso \\
\hline
\end{tabular}

Fonte: Lipschitz (1994). IMC - índice de massa corporal.

A relação cintura/estatura ( $\mathrm{RCE}$ ) foi avaliada dividindo-se a cintura pela estatura, partindo-se da premissa de que a circunferência da cintura de um indivíduo não deve ser maior do que a metade da sua estatura. Os valores de referência, de acordo com Pitanga (2011) são: 0,52 para homens e 0,53 para mulheres. Quando maiores que a referência, indicam risco cardiovascular (PITANGA, 2011). 
A circunferência da cintura foi aferida com uma fita métrica inelástica e flexível da marca Prime $M e d^{\circledR}$ com capacidade de $150 \mathrm{~cm}$, no ponto médio entre a última costela e a crista ilíaca em triplicata, com o indivíduo em posição ereta, abdome relaxado, braços estendidos lateralmente ao longo do corpo com a palma das mãos voltadas para ele, pés um pouco afastados e peso igualmente distribuído entre os dois membros inferiores. Para a classificação da circunferência da cintura foram utilizadas como referências a Sociedade Brasileira De Diabetes (2017). (Tabela 2).

Tabela 2 - Pontos de corte de circunferência da cintura para a definição do risco de complicações metabólicas.

\begin{tabular}{|l|l|l|}
\hline Sexo & \multicolumn{2}{|l|}{ Risco para complicações metabólicas } \\
\cline { 2 - 3 } & Elevado $(\mathbf{c m})$ & Muito elevado $(\mathbf{c m})$ \\
\hline Homens & 94 & $>102$ \\
\hline Mulheres & 80 & $>88$ \\
\hline
\end{tabular}

Fonte: Sociedade Brasileira de Diabetes, 2017

Já a circunferência da panturrilha (CP) foi mensurada a fim de avaliar a perda de massa magra, com o indivíduo sentado, perna flexionada formando um ângulo de $90^{\circ}$ com o joelho, posicionando a fita na circunferência de maior diâmetro da panturrilha e aferida lateralmente. Os valores de referência adotados, que indicaram redução da massa muscular foram: $<33 \mathrm{~cm}$ para mulheres e $<34 \mathrm{~cm}$ para homens (PAGOTTO, 2018).

A circunferência braquial foi mensurada com o indivíduo em posição anatômica. Com braço flexionado formando um ângulo de $90^{\circ}$ com o cotovelo, localizou-se o ponto médio entre o acrômio e a extremidade do olécrano. Após a marcação do ponto médio, o indivíduo estendeu os braços ao longo do corpo com palma da mão voltada para a coxa e a medida da circunferência foi realizada em milímetros. Essa medida foi utilizada para estimativa de tecido muscular, obtida através dos resultados da circunferência do braço e DCT, utilizando as fórmulas adequadas (FRISANCHO, 1990). 
A classificação do percentual de adequação da CMB foi feita da seguinte forma: desnutrição grave $<70 \%$, moderada $>70-80 \%$, leve $>80-90 \%$ e eutrófico $>90-100$ $\%$. Os percentuais para cálculo do \% de adequação foram obtidos em tabelas de referência, de acordo com Frisancho (1990).

Para a determinação da gordura corporal foram medidas as quatros dobras cutâneas utilizando-se um adipômetro de marca $C E S C O R F^{\circledR}$. Dobra cutânea tricipital foi feita com o indivíduo de costas para o medidor, onde foi marcado o ponto médio entre o acrômio e o olécrano, formando a dobra de tecido adiposo (SAMPAIO, 2012).

Dobra cutânea subescapular realizada com o indivíduo em posição ortostática de costas para o medidor, solicitando que o mesmo dobrasse o braço para trás, sobre os arcos costais, formando um ângulo de $90^{\circ}$ com o cotovelo. Com o auxílio dos dedos, localizou-se e marcou-se o ponto abaixo do ângulo inferior da escapula, onde foi feita a medida da dobra (SAMPAIO, 2012).

Dobra cutânea suprailíaca foi medida em posição ortostática, visualizada pelo observador lateralmente. Tomando-se uma dobra cutânea oblíqua, imediatamente acima da crista ilíaca, coincidente com uma linha imaginária descida da linha axilar anterior. $O$ aparelho foi fixado a $1 \mathrm{~cm}$ abaixo da inserção da prega (SAMPAIO, 2012).

A dobra cutânea da panturrilha foi mensurada no ponto de maior perímetro da perna, com indivíduo sentado e a articulação do joelho em flexão formando um ângulo de 90응 com a coxa. O percentual de gordura foi estimado para os valores de IMC específicos para idosos e classificado de acordo com estudo realizado por Gallagher et al. (2000) com 1626 indivíduos de ambos os sexos e de 3 diferentes etnias (GALLAGHER, 2000). (tabela 3).

Tabela 3 - Pontos de corte estimados para percentual de gordura corporal de idosos com 60-79 anos, de acordo com a raça e IMC.

Feminino (\%)
Masculino (\%) 


\begin{tabular}{|l|l|l|l|l|l|l|}
\hline $\begin{array}{l}\text { IMC } \\
\left(\mathrm{Kg} / \mathrm{m}^{2}\right.\end{array}$ & $\begin{array}{l}\text { Afrodescenden } \\
\text { te }\end{array}$ & $\begin{array}{l}\text { Amarel } \\
\mathbf{0}\end{array}$ & $\begin{array}{l}\text { Branc } \\
\mathbf{0}\end{array}$ & $\begin{array}{l}\text { Afrodescenden } \\
\text { te }\end{array}$ & $\begin{array}{l}\text { Amarel } \\
\mathbf{0}\end{array}$ & $\begin{array}{l}\text { Branc } \\
\mathbf{0}\end{array}$ \\
\hline 22 & 27,4 & 30,9 & 29,7 & 13,1 & 16,6 & 15,5 \\
\hline 27 & 37,8 & 38,9 & 41,0 & 24,8 & 25,9 & 27,0 \\
\hline
\end{tabular}

Fonte: adaptado de Gallagher et al., 2000. IMC - Índice de Massa Corporal.

Para os cálculos foi aplicada a equação de predição de densidade corporal (DC) proposta por Petroski (1995), e a fórmula de Siri, que utiliza a DC para cálculo do percentual de gordura (PETROSKI, 1995).

\subsection{CONSUMO ALIMENTAR}

A avaliação do consumo alimentar foi feita pela análise de 3 registros alimentares. $O$ voluntário escolheu 3 dias da semana, não consecutivos, incluindo um dia de final de semana, sendo segunda-feira, quarta-feira e sábado e registrou todos os alimentos consumidos com suas respectivas quantidades, medidas caseiras, marcas comerciais, formas de preparação, horário e local das refeições. Posteriormente, esses registros foram analisados individualmente para a avaliação do consumo alimentar de forma quantitativa. Para analisar a adequação de carboidratos, proteínas, lipídios e micronutrientes (cálcio, vitamina $\mathrm{D}$, ferro e sódio) foram utilizados os valores de referência para a idade média da amostra, considerando o sexo, de acordo com as DRIs (Dietary Reference Intake), (1997) (INSTITUTE OF MEDICINE, 1997; PADOVANI, 2006).

\subsection{TRATAMENTO DOS DADOS}

Os dados foram representados de forma descritiva. Para as variáveis contínuas, utilizou-se a média aritmética, com seu respectivo desvio padrão (DP), e para as variáveis categóricas, a frequência absoluta $(\mathrm{N})$ e o percentual (\%). A tabulação e cálculo dos dados descritivos foram elaborados utilizando-se o programa Microsoft Office Excel, versão 2013. 


\section{RESULTADOS}

O programa de saúde, inicialmente possuía 82 participantes cadastrados e destes, 60 eram frequentadores assíduos e concordaram em participar do estudo. Dos 60 entrevistados, 6 tinham menos de 60 anos e foram, portanto, excluídos. Participaram 54 idosos, sendo a maioria do sexo feminino (85\%), com idade média de 72,4 $\pm 6,6$ anos, mínima de 60 e máxima de 85 anos. Os idosos eram, em sua maioria, caucasianos, casados ou em união estável e aposentados (tabela 4).

Analisando o estado nutricional, o IMC indicou eutrofia para homens $(\mathrm{IMC}=26,0$ $\mathrm{kg} / \mathrm{m}^{2}$ ) e excesso de peso para mulheres $\left(\mathrm{IMC}=27,7 \mathrm{~kg} / \mathrm{m}^{2}\right)$. Além disso, $50 \%$ de todos os idosos apresentaram excesso de peso. A circunferência da cintura indicou risco para doenças cardiovasculares entre mulheres, mas não entre homens. A CMB mostrou-se adequada para $100 \%$ das idosas avaliadas e para a maioria dos homens $(87,5 \%)$. Porém, a circunferência da panturrilha indicou massa magra adequada somente para $56,5 \%$ das mulheres e $37,5 \%$ dos homens. O percentual de gordura esteve adequado para mulheres, porém, aumentado para homens ( $M$ : média $=33,3 \%$, $\mathrm{DP}= \pm 5,6 \% ; \mathrm{H}$ : média $=31,2 \%, \mathrm{DP}= \pm 6,9 \%$ ). Na tabela 4, são apresentadas as características da amostra. 
Tabela 4 - Dados sócio demográficos e antropométricos da amostra, de acordo com o sexo.

\begin{tabular}{|l|l|l|}
\hline Variáveis & Masculino & Feminino \\
\hline Idade (Anos) & $\mathrm{N}=8$ & $\mathrm{~N}=46$ \\
\hline Caucasianos & $76 \pm 8$ & $71 \pm 6$ \\
\hline Casados ou união estável & $6(75,0)$ & $33(71,7)$ \\
\hline Até ensino fundamental completo & $8(100)$ & $25(54,3)$ \\
\hline Aposentados & $3(37,5)$ & $32(69,6)$ \\
\hline Etilistas & $8(100)$ & $37(80,4)$ \\
\hline Tabagistas & $2(25,0)$ & $5(10,9)$ \\
\hline Diabéticos & - & $1(2,2)$ \\
\hline Hipertensos & $2(25,0)$ & $10(21,7)$ \\
\hline Índice Massa Corporal (kg/m²) & $2(25,0)$ & $30(65,0)$ \\
\hline Razão Cintura Estatura & $26,0 \pm 5,6$ & $27,7 \pm 4,3$ \\
\hline Circunferência da Cintura (cm) & $0,55 \pm 0,07$ & $0,50 \pm 0,1$ \\
\hline Acima de 90\% de adequação da CMB & $9(87,5)$ & $46(100)$ \\
\hline CP adequada & $3(37,5)$ & $26(56,5)$ \\
\hline Gordura (\%) Corporal & $31,2 \pm 7$ & $33,3 \pm 5,6$ \\
\hline
\end{tabular}

Valores representados em média \pm desvio padrão ou n (\%). CMB - Circunferência Muscular do Braço. CP - Circunferência da Panturrilha. Fonte: autor.

Os registros alimentares foram avaliados em 34 (63\%) dos indivíduos que retornaram os registros alimentares completos e com informações válidas. A ingestão calórica média da amostra avaliada esteve abaixo do recomendado pelas DRIs, tanto para 
homens (2.497 kcal para um homem de 76 anos) quanto para mulheres $(2.039 \mathrm{kcal}$ para uma mulher de 71 anos).

Já a ingestão de carboidratos esteve adequada para ambos os sexos, de acordo com a recomendação (45-65\%). Da mesma forma, a ingestão de proteínas e lipídeos também esteve adequada para homens e mulheres (recomendação: 10-35\% e 20$35 \%$, respectivamente).

Quanto aos micronutrientes, a ingestão de cálcio média encontrada (recomendação: $1200 \mathrm{mg}, 70$ anos) e vitamina $\mathrm{D}$ (recomendação: $15 \mu \mathrm{g}, 70$ anos) estavam inadequadas para ambos os sexos. Em contrapartida, a ingestão média de ferro esteve adequada para os homens e mulheres (recomendação: $8 \mathrm{mg}, 70$ anos). Já a média de ingestão de sódio esteve adequada em ambos os sexos, considerando que se recomenda a ingestão menor que $2400 \mathrm{mg}$ por dia em qualquer idade (Tabela 5).

Tabela 5: Médias e desvios padrão da ingestão de macro e micronutrientes avaliados pelo registro alimentar de 3 dias, de acordo com o sexo.

\begin{tabular}{|c|c|c|c|c|}
\hline \multirow[t]{2}{*}{ Variáveis } & \multirow{2}{*}{$\begin{array}{l}\text { Masculino } \\
\text { Média } \pm \text { Dp }\end{array}$} & \multirow{2}{*}{$\begin{array}{l}\text { Feminino } \\
\text { Média } \pm \text { Dp }\end{array}$} & Masculino & Feminino \\
\hline & & & \multicolumn{2}{|c|}{ Recomendação } \\
\hline Calorias totais (Kcal) & $1340 \pm 429,7$ & $1194 \pm 333,4$ & 2497 & 2039 \\
\hline Carboidratos (\%) & $47,4 \pm 11,5$ & $51,9 \pm 7,5$ & \multicolumn{2}{|l|}{$45-65$} \\
\hline Proteínas (\%) & $21,5 \pm 7,5$ & $22,3 \pm 5,9$ & \multicolumn{2}{|l|}{$10-35$} \\
\hline Lipídeos (\%) & $29 \pm 6,7$ & $26,9 \pm 5$ & \multicolumn{2}{|l|}{$20-35$} \\
\hline Cálcio (mg) & $460,3 \pm 216,4$ & $326 \pm 193$ & \multicolumn{2}{|l|}{1200} \\
\hline Ferro (mg) & $12,4 \pm 3,3$ & $9 \pm 3,4$ & \multicolumn{2}{|l|}{8} \\
\hline Vitamina D $(\mu \mathrm{g})$ & $11,8 \pm 23$ & $1 \pm 0,9$ & \multicolumn{2}{|l|}{15} \\
\hline Sódio (mg) & $2372,7 \pm 1864,4$ & $1216,2 \pm 753,7$ & \multicolumn{2}{|l|}{$<2400$} \\
\hline
\end{tabular}

Valores representados em média \pm desvio padrão ou $n(\%)$. Fonte: autor.

\section{DISCUSSÃO}


Este estudo descreveu a avaliação antropométrica e alimentar de idosos participantes de um programa de saúde. O grupo constituiu-se, na sua maior parte, por idosos do sexo feminino (85\%), assim como na população brasileira, de modo geral, em que as mulheres mostram maior interesse na procura pela saúde. Isso pode estar relacionado ao maior alcance dos programas de saúde, que geralmente focam mais na saúde da mulher, crianças e idosos, na maioria das vezes acompanhados por mulheres (SOUZA, 2013).

A média de idade encontrada foi de 72,4 anos, equivalente a outro estudo, que verificou média de 70,4 anos de idade (BORBA; MUNIZ, 2011).

Com relação à escolaridade, percebeu-se que, grande parte dos entrevistados teve pouco acesso à educação, não ultrapassando o Ensino Fundamental (68,5\%) e isso pode repercutir de maneira negativa na assistência e na qualidade de vida do idoso. Além disso, pode implicar na dificuldade de entendimento e aprendizagem relacionados a hábitos alimentares saudáveis (BORBA; MUNIZ, 2011; SILVA; SIMÕES; LEITE, 2007).

O estudo em questão mostrou baixo número de etilistas e tabagistas, resultado relevante para a saúde dessa população. Em contrapartida, um estudo observou maior prevalência de idosos que consumiam álcool e cigarros, sendo $20,8 \%$ dos homens e $11,7 \%$ das mulheres. Alcoolismo e tabagismo são hábitos que podem trazer consequências nocivas à saúde do idoso, visto que o tabaco é considerado um dos mais influentes agentes carcinogênicos para o ser humano. Já o consumo elevado de álcool está associado à hipertensão arterial, cirrose, acidente vascular hemorrágico e aos cânceres da orofaringe, laringe, esôfago e fígado (SENGER, 2011).

A média de IMC encontrada mostrou excesso de peso para a maioria das mulheres $\left(27,7 \mathrm{~kg} / \mathrm{m}^{2}\right)$, valor semelhante ao estudo de Closs (2015), onde mulheres mostraram níveis elevados de IMC $(29,58 \pm 5,96 \mathrm{~kg} / \mathrm{m} 2)$. A elevação o IMC pode estar associada a risco aumentado de morte, além de associação ao desenvolvimento de fatores de risco cardiovasculares em idosos (CLOSS, 2015). 
Contudo, comparando-se esta pesquisa com outros estudos que também avaliaram o IMC para essa faixa etária, indicando excesso de peso para ambos os sexos, relatam a relação com o estilo de vida. Sendo assim, a elevação do IMC está associada a causas de mortalidade entre homens e mulheres com idade superior a 60 anos. Além disso, tanto o aumento de massa gorda como a perda de massa magra estão associados ao risco e desenvolvimento de doenças. Vale ressaltar que o IMC é uma variável que não deve ser avaliada individualmente por ser um método que não considera as repartições do corpo separadamente. Sendo assim, é aconselhável associá-los com outros indicadores como dobras e circunferências (SANADA, 2018).

A Relação Cintura e Estatura (RCE) encontrada na investigação indicou risco para doenças cardiovasculares entre homens, corroborando com o estudo realizado por Closs (2015), com idosos, que encontrou RCE > 0,5. Utilizando-se a RCE para avaliar o risco cardiovascular, considera-se que a circunferência da cintura não deve ser maior do que a metade da estatura, indicando obesidade central (CLOSS, 2015). Este método vem sendo bastante citado na literatura como indicador de risco nos idosos, além da circunferência da cintura (CC) isoladamente, por ser utilizado para avaliar risco coronariano elevado e tem relação com a mortalidade (PITANGA, 2011). A RCE representa um dos melhores indicadores de risco em relação a outros métodos de identificação para doenças cardiovasculares, como por exemplo CC e Relação Cintura e Quadril (RCQ) (BENEDETTI; MEURER; MORINI, 2012).

Neste estudo, a CC mostrou adequação para o sexo masculino e inadequação para o feminino, assim como no estudo de Machado; Coelho e Coelho (2010) em que a CC foi de $87,23 \pm 14,2 \mathrm{~cm}$ para o sexo feminino. Segundo a Sociedade Brasileira de Diabetes (2017) sabe-se que o acúmulo de gordura na região abdominal tem sido usado para identificação de adiposidade visceral associado ao risco de doenças cardiovasculares e metabólicas. (SOCIEDADE BRASILEIRA DE DIABETES, 2017).

Alguns estudos mostram que os homens tendem a ter maiores depósitos de massa magra em relação às mulheres. Porém, nesta pesquisa, 100\% das mulheres mostraram adequação maior que $90 \%$ à CMB. Diferente dos resultados apresentados 
no estudo feito por Silva et al., (2015), a CMB esteve semelhante para ambos os sexos $(H: 23,8 \pm 4,1$ e $M: 23,5 \pm 4,2 ; p=0,16)$.

A CP, por outro lado, mostrou-se inadequada para uma parcela considerável dos idosos avaliados neste estudo, indicando possível prejuízo da massa magra. Ao contrário, do estudo de Closs (2015) que encontrou resultado satisfatório, mostrando preservação da massa muscular. A CP adequada tem sido associada com maior força muscular, melhor desempenho físico e funcional, além de ser indicada como a mais sensível para avaliar a massa muscular em idosos (CLOSS, 2015).

Os resultados do percentual de gordura desta pesquisa indicaram excesso de gordura subcutânea nos homens, corroborando com o percentual de gordura encontrado no estudo que avaliou 395 idosos residentes em instituições asilares do Rio de Janeiro. As medidas de dobras cutâneas vêm sendo bastante utilizadas na estimativa da gordura corporal, por serem consideradas de fácil execução, de baixo custo e aplicáveis em estudos de campo e epidemiológicos (MACHADO; COELHO; COELHO, 2010).

Apesar de eutróficos, os homens do grupo de idosos apresentaram percentual de gordura elevado, o que pode indicar risco aumentado para DCNT. O acúmulo de gordura corporal pode acometer diferentes sistemas, e está relacionado a problemas metabólicos e sanguíneos, além de tornar o idoso mais vulnerável a limitações e redução na atividade de vida diária (MIRANDA, 2017). Além disso, a maioria dos homens avaliados apresentavam a massa magra prejudicada, mesmo sendo integrantes de um programa que oferece atividade física duas vezes por semana. Em um estudo que analisou a composição corporal de 37 idosos, no Rio de Janeiro e empregou vários métodos para o cálculo do percentual de gordura, observou-se que um dos métodos que mostrou resultado significativo foi por medida de 4 dobras, a mesma deste estudo, tendo como referência Petroski, (1995).

A avaliação da ingestão de macronutrientes e micronutrientes foi estimada segundo sexo e grupo etário (51-70 anos; > 70 anos), utilizando as DRls (Dietary Referece Intakes) (PADOVANI, 2006). Apesar de 50\% dos integrantes estarem com excesso 
de peso, pode-se perceber a ingestão calórica inadequada de acordo com a recomendação, sendo que a média da necessidade energética diária desses indivíduos é de aproximadamente 2497 e 2039 Kcal (INSTITUTE OF MEDICINE, 1997). Isso significa que os idosos consomem menos calorias do que a necessidade diária. Valores semelhantes foram observados no estudo de Venturini et al., (2015), que encontrou valores entre 1320 e $1564 \mathrm{Kcal}$. Apesar disso, nesta pesquisa, a proporção de macronutrientes ingeridos (carboidratos, proteínas e lipídeos) mostrouse adequada. Principalmente para os idosos, é importante que a dieta esteja balanceada em relação aos macronutrientes, assim como foi observado (VENTURINI, 2015).

No entanto, foram observadas inadequações na ingestão de micronutrientes como o cálcio e vitamina $\mathrm{D}$, semelhante à Pesquisa de Orçamentos Familiares (POF) 20082009 em idosos no Brasil, que encontrou prevalências de inadequação próximas de 80\%. Segundo Leite; Baratto e Silva (2014), o cálcio é de extrema importância no processo de envelhecimento, pois auxilia na prevenção de osteoporose e é um ingrediente dietético importante para o tecido ósseo (FISBERG et al., 2014). Seu papel no organismo depende da ação da vitamina $\mathrm{D}$, juntamente com a exposição ao sol $\mathrm{e}$ prática de atividades físicas, tornando-se importantes protetores contra a perda de massa óssea e o desenvolvimento da osteoporose. Sua recomendação diária é de $1200 \mathrm{mg}$ por dia a partir dos 51 anos de idade para homens e mulheres justamente por esse grupo ser mais vulnerável à osteoporose (INSTITUTE OF MEDICINE, 1997). Ainda segundo a POF, a vitamina D apresentou ingestão próxima de $100 \%$ de adequação em todas as regiões, diferente desta pesquisa que encontrou inadequação para ambos os sexos (VENTURINI, 2015).

O ferro foi o único micronutriente que esteve com ingestão adequada, com média de $12,4 \mathrm{mg}$ para os homens e $9 \mathrm{mg}$ para as mulheres. Ao contrário, do estudo de Venturini et al., (2015) que mostrou que as mulheres costumam consumir menos ferro do que os homens. De acordo com Gualandro; Hojaij e Filho (2010) a deficiência de ferro é considerada comum entre os idosos. Sua causa pode ser tanto pela ingestão de medicamentos que prejudicam sua absorção, quanto a ingestão inadequada dos 
alimentos fontes como as carnes, feijão, frutas e vegetais. Aproximadamente $150 \mathrm{~g}$ de carne atingem praticamente metade da recomendação diária, portanto é considerado um micronutriente de fácil aquisição (PFRIMER; FERRIOLLI, 2008). Estudos apontam que a anemia é um distúrbio frequente na população idosa e que sua prevalência cresce com a idade. É considerada um fator de risco para morbidade e mortalidade, além de interferir no desempenho físico e mental e na habilidade de manter as atividades diárias, afetando a qualidade de vida dessa população, portanto, o consumo de alimentos fontes é fundamental. De acordo com as DRIs, os valores de referência para ingestão do ferro são 8,0 mg/dia para mulheres e homens (NATIONAL ACADEMY OF SCIENCES, 2001).

Com o processo de envelhecimento, o idoso tende a se tornar bem mais vulnerável a carências nutricionais, em especial a de vitaminas e minerais, principalmente com o tecido ósseo, predispondo a osteoporose ou fraturas ósseas. Sendo assim, o idoso necessita de um plano alimentar específico e adequado para suas particularidades nutricionais (VENTURINI, 2015; COUSSIRAT, 2012).

Esta pesquisa representou uma investigação de grande relevância para a população em questão. Porém, algumas limitações devem ser pontuadas. A primeira, relacionada ao tamanho da amostra, que pode ser considerada pequena. Mesmo assim, 66\% dos idosos matriculados no grupo foram avaliados. Outro ponto limitante foi o método utilizado para avaliar a ingestão alimentar (registro alimentar de 3 dias). Não se pode assegurar que o consumo alimentar de apenas 3 dias reflete com precisão o hábito alimentar do indivíduo, além do fato de que podem haver propositalmente modificações no habito alimentar durante o período de avaliação. A avaliação do consumo alimentar pode ser influenciada por vários fatores, como a interferência na qualidade da informação, nível educacional, a preocupação com a aprovação social e padrão de beleza e a própria percepção do indivíduo sobre a alimentação saudável (MENEZES, 2013).

\section{CONCLUSÃO}


O presente estudo encontrou percentual de gordura elevado para o sexo masculino, IMC e circunferência da cintura elevada para as mulheres, podendo implicar no surgimento das DCNT. Esse fato chamou a atenção para a necessidade de uma orientação nutricional, para despertar a adoção de hábitos alimentares mais saudáveis, pois é fato inevitável a relação entre esses hábitos de vida com os dados antropométricos da população, em especial dos idosos, que já são atingidos com inúmeras influências fisiológicas do avançar da idade.

Foram também observadas importantes deficiências nutricionais na população de idosos estudada, principalmente em micronutrientes. Acredita-se que esta possa ser a realidade de outras populações com características semelhantes, o que mostra a importância de novos estudos com essa faixa etária.

Sendo assim, é de suma importância detectar os indivíduos em risco nutricional precocemente, pois assim torna-se possível realizar uma intervenção nutricional primária e mais específica, como um desenvolvimento de ações preventivas, orientações nutricionais na atenção básica de saúde, visando à promoção da alimentação saudável e estímulo a atividade física, a fim de promover melhorias na qualidade de vida dos idosos.

Portanto, a ampliação de estudos de composição corporal e perfil antropométrico em idosos, utilizando as dobras cutâneas e perímetros musculares de modo mais abrangentes favorecem o melhor diagnóstico do estado nutricional dessa população, o que facilita o planejamento e a elaboração de uma conduta a ser aplicada. Sendo assim, torna-se cada vez mais importante o conhecimento específico das características antropométricas e alimentares de cada grupo etário, possibilitando a construção de padrões locais de referência. Diante disso, sugere-se a realização de mais pesquisas com esse grupo a fim de contribuir para um envelhecimento natural e saudável.

\section{REFERÊNCIAS}


BENEDETTI, T. R. B.; MEURER, S. T.; MORINI, S. Índices antropométricos relacionados a doenças cardiovasculares e metabólicas em idosos. Rev. Educ. Fís/UEM. Florianópolis-SC, v. 23, n. 1, p. 123-130, 1. trim. 2012.

BORBA, T. B.; MUNIZ, R. M. Sobrepeso em idosos hipertensos e diabéticos cadastrados no Sistema HiperDia da Unidade Básica de Saúde do Simões Lopes, Pelotas, RS, Brasil. Rev. Enferm. Saúde. v.1, n. 1, p. 69-76, 2011.

CHUMLEA, W. C, ROCHE, A. F, STEINBAUGH M. L. Estimating Stature from Knee Height for Persons 60 to 90 Years of Age. Journal of the American Geriatrics Society. V. 33, n. 2, p. 116-120, fev, 1985.

CLOSS, V. E.; ROSEMBERG, L.S.; ETTRICH, B. G.; GOMES, I.; SCHWANKE, C. H. Medidas antropométricas em idosos assistidos na atenção básica e sua associação com gênero, idade e síndrome da fragilidade: dados do EMI-SUS. Vev.Scientia Medica. v. 25, n. 3, p. 1-7, 2015.

COUSSIRAT, C.; BATISTA, C.; SCHNEIDER, R. H.; RESENDE, T.; SCHWANKE C. H. A. Vitaminas B12, B6, B9 e homocisteína e sua relação com a massa óssea em idosos. Rev Brasil de Geriat e Gerontol. v.15, n. 3, p. 577-585, 2012.

FISBERG, R. M.; MARCHIONI, D. M.; CASTRO, M. A.; JUNIOR, E. V.; ARAÚJO, M. C.; BEZERRA, I. N.; PEREIRA, R. A.; SICHIERI, R. Ingestão inadequada de nutrientes na população de idosos do Brasil: Inquérito Nacional de Alimentação 2008-2009. Rev Saúde Pública. v. 47 n. 1 Supl):222S-30S 2013.

FRISANCHO, A. R. Anthropometric standards for the assessment of growth and nutritional status. Ann Arbor: The University Academic Press, P. 48-53. 1990.

GALLAGHER, D.; HEYMSFIELD, S. B.; HEO, M.; JEBB, S. A.; MURGATROYD. R. A.; SAKAMOTO, Y. Healthy percentage body fat ranges: an approach for developing guidelines based on body mass index. Am J Clin Nutr. EUA, v. 72, p. 694-701, 2000. 
GEUS, L. M. M.; MACIEL, C. S.; BURDA, I. C. A DAROS, S.J.; BATISTEL, S.; MARTINS, T.C. A.; FERREIRA, V. A; DITTERICH, R. G. A importância na inserção do nutricionista na Estratégia Saúde da Família. Ciência e Saúde Coletiva, Ponta Grossa Pr, v. 16, n.1, p. 119-23, dez. / 2011.

GUALANDRO, S. F. M.; HOJAIJ, N. H. S. L.; FILHO, W. J. Deficiência de ferro no idoso. Rev Bras Hematol Hemoter. São Paulo, v. 32, n. 2, p. 57-61, 2010.

INSTITUTE OF MEDICINE. Dietary reference intakes for calcium, phosphorus, magnesium, vitamin D, and fluoride. Washington (DC): National Academy Press; 1997.

INSTITUTO BRASILEIRO DE GEOGRAFIA E ESTATÍSTICA (IBGE). População de idosos vai triplicar até 2050 no Brasil, revela pesquisa São Paulo: 2016. Disponível em:

$<$ https://www.correiobraziliense.com.br/app/noticia/economia/2016/08/30/internas_ec onomia,546485/populacao-idosa-vai-triplicar-ate-2050-revela-pesquisa.shtml< Acesso em 05 mar. 2019.

LEITE, S. C.; BARATTO, I.; SILVA, R. Consumo de cálcio e risco de osteoporose em uma população de idosos. Rev Bras de Obes, Nutriç e Emagrec. v. 8, n. 48, P. 165174, 2014.

LIPSCHITZ, D. A. Screening for nutritional status in the elderly. Nutrition in Old Age, v. 21 , n. 1, p. 55-67, 1994.

MACHADO, R. S. P.; COELHO, M. A. S. C.; COELHO, K. S. C. Percentual de gordura corporal em idosos: comparação entre os métodos de estimativa pela área adiposa do braço, pela dobra cutânea tricipital e por bioimpedância tetrapolar. Rev. Bras. Geriatr. Gerontol. v. 13, n. 1, p. 17-27, 2010.

MARCHIONI, D. M. L.; FISBERG, R. M. Dieta, nutrição e prevenção de doenças crônicas não transmissíveis. In: CUPPARI, L. Nutrição nas doenças crônicas nãotransmissíveis. 1. Ed. São Paulo, SP. Manole, Cap. 1. P. 2-24, 2009. 
MELlO, A. C.; CARVAlHO, M. S. AlVES, L. C., GOMES, V. P.; ENGSTROM, E. M. Consumo alimentar e antropometria relacionados à síndrome de fragilidade em idosos residentes em comunidade de baixa renda de um grande centro urbano. Cadernos de Saúde Pública. Rio de Janeiro, v.33, n. 8, 2017.

MENEZES, N.; BRITO, T. T.; ARAÚJO, M. B. P.; SILVA, T. M.; NOLASCO, C. C. N.; RENNARLI, R.; FISCHE, A. T. S. Perfil antropométrico dos idosos residentes em Campina Grande-PB. Rev Bras de Geriat e Geront. V. 16, n. 1, p. 19-27, 2013.

MIRANDA, G. M. D.; MENDES, A. C. G.; SILVA, A. L. A.; O envelhecimento populacional brasileiro: desafios e consequências sociais atuais e futuras. Rev. bras. Geriatria. Gerontol. Rio de Janeiro, v. 19, n. 3, p. 507-519, jun. 2016.

MIRANDA, R. N. A.; CARVALHO, E. P.; AMORIM, I. R.; SANTOS, K. S.; SERRÃO, F. O. Conhecendo a saúde nutricional de idosos atendidos em uma organização não governamental, Benevides/pa. Rev. Conexão UEPG. v. 13, n. 3, p. 3-18, 2017.

MOREIRA, A. J.; NICASTRO, H.; CORDEIRO, R. C.; COIMBRA, P.; SILVIA FRANGELLA, V. S. Composição corporal de idosos segundo a antropometria. Rev Brasil de Geront. v. 12, n. 2, p. 201-213. 2009.

NATIONAL ACADEMY OF SCIENCES. Dietary Reference Intakes for Vitamin A, Vitamin K, Arsenic, Boron, Chromium, Copper, lodine, Iron, Manganese, Molybdenum, Nickel, Silicon, Vanadium, and Zinc. Washington DC: National Academy Press, 2001.

NETO, L. S. S.; KARNIKOWISKI, M. G.; TAVARES, A. B.; LIMA, R. M. Associação entre sarcopenia, obesidade sarcopênica e força muscular com variáveis relacionadas de qualidade de vida em idosas. Rev Bras Fisioter. Palmas To, v. 16, n. 5, 360-7. Fev. /abr. 2012.

PADOVANI, R. M.; AMAYA-FARFÁN, J.; COLUGNATI, F. A. B.; DOMENE, S. M. Á. Dietary reference intakes: aplicabilidade das tabelas em estudos nutricionais. Rev de Nutri. v.19 n. 6, p. 741-760, 2006. 
PAGOTTO, V.; SANTOS, K. F. MALAQUIAS, S. G.; BACHION, M. M.; SILVEIRA, E. A. Circunferência da panturrilha: validação clínica para avaliação de massa muscular em idosos. Rev Bras Enferm. Goiânia-GO, v. 71, n. 2, p. 343-50, mar./jun.2018

PETROSKI, EL. Desenvolvimento e validação de equações generalizadas para a estimativa da densidade corporal em adultos. Santa Maria: Universidade Federal de Santa Maria, 1995.

PFRIMER, K.; FERRIOLLI, E. Avaliação Nutricional do Idoso. In: VITOLO, M. R. Nutrição da Gestação ao Envelhecimento. 1. ed. Rio de Janeiro. Rubio Ltda, 2008. Cap. 45, p. 435-449.

PITANGA, F. J. G. Antropometria na avaliação da obesidade abdominal e risco coronariano. Rev Bras Cineantropom Desempenho Hum. Salvador BA, v. 13, n. 1, p. 238-241, nov./dez.2011.

SAMPAIO, L. R.; SILVA, M. C. M.; OLIVEIRA, T. M.; RAMOS, C. I. Avaliação Nutricional: ed. 1 reimpressão Salvador: Edufba, 2012.

SANADA, K.; CHEN, R.; WILLCOX, B.; OHARA, T.; WEN, A.; TAKENAKA, C.; MASAKI, K. Association of sarcopenic obesity predicted by anthropometric measurements and 24-y all-cause mortality in elderly men: The Kuakini Honolulu Heart Program. Nutrition. Japão, v. 46, p. 97-102, Abr./set. 2018.

SENGER, A. E. V.; ELY, L. S.; GANDOLFI, T.; SCHNEIDER, R. H.; GOMES. I.; CARLI G. A. Alcoolismo e tabagismo em idosos: relação com ingestão alimentar e aspectos socioeconômicos. Rev. Bras. Geriatr. Gerontol. v. 14, n. 4, p. 713-719, 2011.

SILVA, A. L. S. C.; SILVA, B. S.; BRANDÃO, J. M.; BARROSO, S. G.; ROCHA, G. S. Avaliação antropométrica de idosos atendidos no Ambulatório de Nutrição do Centro de Referência em Assistência à Saúde do Idoso da Universidade Federal Fluminense, no município de Niterói-RJ. Demetra 10, n. 2, p. 361-374, 2015. 
SILVA, R. C. P.; SIMÕES, M. J. S.; LEITE, A. A. Fatores de risco para doenças cardiovasculares em Sociedade Brasileira de Diabetes. Diretrizes da sociedade brasileira de diabetes. 1. ed. São Paulo: Clannad, 2017.

SOUZA, R.; FRAGA, J. S.; GOTTSCHALL, A. B. A.; BUSNELLO, F. M.; RABITO, E. I. Avaliação antropométrica em idosos: estimativas de peso e altura e concordância entre classificações de IMC. Rev. Bras. Geriatr. Gerontol. v. 16, n. 1, p. 81-90, 2013. VentuRINI, C. D.; ENGROFF, P.; GOMES, I.; CARLI, G. A. Prevalência de obesidade associada à ingestão calórica, glicemia e perfil lipídico em uma amostra populacional de idosos do Sul do Brasil. Revista Brasileira de Geriatria e Gerontologia. Rio de Janeiro, v. 16, n. 3, p. 591-601, jul. 2013.

Enviado: Março, 2021.

Aprovado: Junho, 2021. 